Matsumoto et al. Transcorneal three-Port vitrectomy without conjunctival incision

\title{
Transcorneal three-port vitrectomy without conjunctival incision
}

Abbreviated title: Transcorneal three-port vitrectomy

Makiko Matsumoto, MD, Kiyoshi Suzuma, MD, PhD, Noritake Miyamura, MD, PhD,

Eiko Tsuiki, MD, PhD, Takashi Kitaoka, MD, PhD

Correspondence and requests for reprints to: Makiko Matsumoto, MD

Department of Ophthalmology and Visual Sciences, Graduate School of Biomedical

Sciences, Nagasaki University, 1-7-1 Sakamoto, Nagasaki 852-8501, Japan (Fax number: 011-8195-819-7347, Telephone number: 011-8195-819-7345, E-mail address: makimaki@nagasaki-u.ac.jp)

Proprietary interest: none

We did not receive any financial support for this study. 
Matsumoto et al. Transcorneal three-Port vitrectomy without conjunctival incision

Key Words: epiretinal membrane, filtering bleb, glaucoma, 25-gauge-system

Brief summary statement: We treated epiretinal membrane (ERM) using 25-gauge transcorneal vitrectomy without a conjunctival incision. The conjunctiva and ocular surface remained intact. This approach is suitable especially for treating glaucoma with a filtering bleb. (32 words/50 words or less) 
Matsumoto et al. Transcorneal three-Port vitrectomy without conjunctival incision

\section{Abstract}

Background: Although widely accepted, pars plana vitrectomy is difficult to perform when patients have a large filtering bleb. We describe technical details of 25-gauge transcorneal vitrectomy and the clinical outcomes.

Methods: We performed 25-gauge transcorneal vitrectomy on seven eyes of seven patients (female: male, 4:3; age, 39 to 77 years; mean, 66.1 years) between January 2005 and February 2007. Phacoemulsification via a corneal incision was followed by continuous curvilinear capsulorrhexis (CCC) of the posterior capsule. We used a notched contact lens to perform 25-gauge transcorneal vitrectomy.

Results: Four patients with idiopathic ERM and three with secondary ERM were treated by 25-gauge vitrectomy. Postoperative visual acuity was good in most of them and the cell density of the corneal endothelium was maintained.

Conclusion: These advantages of this procedure are that the conjunctiva and ocular surface can be completely maintained and vitreous incarceration can be avoided. Thus, 25-gauge vitrectomy might be particularly suitable for treating glaucoma with a filtering bleb. (158 words/200 words) 
Matsumoto et al. Transcorneal three-Port vitrectomy without conjunctival incision

Introduction: Although widely accepted, pars plana vitrectomy is difficult to perform when patients have a filtering bleb, and vitreous incarceration to a sclera wound could result in retinal detachment. We have performed several 25-gauge transcorneal vitrectomies without a conjunctival incision to treat epiretinal membrane (ERM). We describe the technical details of our procedure and the clinical outcomes.

Patients and methods: We treated ERM in seven eyes of seven patients (female: male, 4:3; age, 39 to 77 years; mean, 66.1 years) between January 2005 and February 2007. A giant bleb developed in one patient after trabeculectomy. Phacoemulsification with a corneal incision was followed by continuous curvilinear capsulorrhexis (CCC) of the posterior capsule (Figure 1A). Three side port limbal incisions were cut using a 23-gauge-V-lance at 9.30, 2.30 and 4 or 8 (at a temporal lower site) o'clock. We also performed 25-gauge transcorneal vitrectomy using a notched contact lens (Figure 1B) (which we made three cuts at the edge of a disposable silicon lens, using a straight knife) core vitrectomy and ERM peeling (Figure 1C). We inserted the intraocular lens into the capsular bag after vitrectomy (Figure 1D).

Results: The clinical summaries for the seven patients are presented in Table 1. A 72-year-old female (Case 1) developed ERM that was discovered 20 years after surgery for retinal detachment. A 39-year-old male (Case 2) who developed a giant bleb after trabeculectomy had been administered with intravitreal triamcinolone acetonide twice for macular edema due to branch retinal vein occlusion, and followed by vitrectomy for ERM and macular edema. Although visual acuity improved from 20/80 to 20/40 two months after the operation, macular edema recurred and visual acuity decreased. A 62-year-old female (Case 3) presented with ERM was accompanied by a retinal tear. Cases 4-7 had idiopathic ERM and Case 6 had a history of medical treatment for 
Matsumoto et al. Transcorneal three-Port vitrectomy without conjunctival incision

glaucoma. An iatrogenic lamellar macular hole discovered in this patient, while peeling the internal limiting membrane during vitrectomy, was treated using SF6 gas tamponade. The defect in the glaucomatous visual field gradually progressed to involve the central visual field. Postoperative visual acuity was good in all patients except for Cases 2 and 6 (Cases 1-7: 20/20, 20/100, 20/16, 20/25, 20/25, 20/100 and 20/20, respectively; Snellen method at 6 months after surgery). The cell density of the corneal endothelium was maintained after transcorneal vitrectomy (Cases 1 and 2, not done; Cases 3-7, mean density before and after surgery: $2734 \pm 348$ and $2569 \pm 99$ (SD) cells $/ \mathrm{mm}^{2}$ respectively; mean decrease in cell density, $165 \pm 304$ (SD) cells/mm²).

Discussion: We satisfactorily treated ERM using 25-gauge transcorneal vitrectomy. The advantages of this procedure are that the conjunctiva and ocular surface can be completely maintained, and vitreous confinement can be avoided. The disadvantages are that both hands are closer to the eye during the procedure and it is more restrictive than standard pars plana vitrectomy. The usual forceps may not easily reach a long axis, and the cornea can be distorted leading to decreased visibility. Peripheral vitrectomy is technically difficult and contraction of the remaining vitreous body could result in retinal detachment. Patients should therefore be followed up for several years. However, we believe that this procedure is suitable, particularly for treating glaucoma with a filtering bleb.

Conclusion: For some conditions such as idiopathic ERM with giant bleb, 25-gauge transcorneal vitrectomy may be a very useful treatment option. 
Matsumoto et al. Transcorneal three-Port vitrectomy without conjunctival incision

\section{Figure legends}

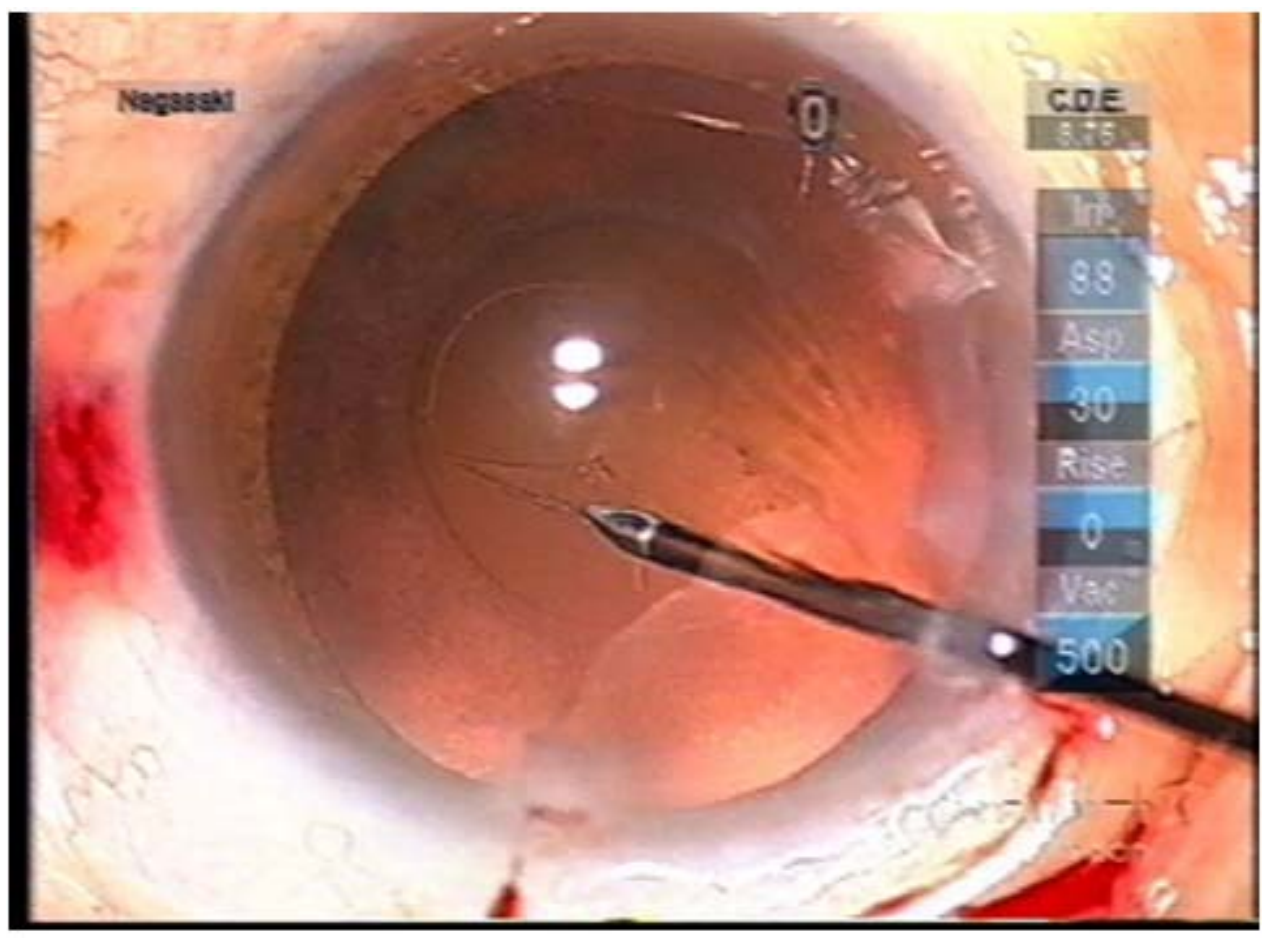

Figure 1A. Continuous curvilinear capsulorrhexis (CCC) of posterior capsule.

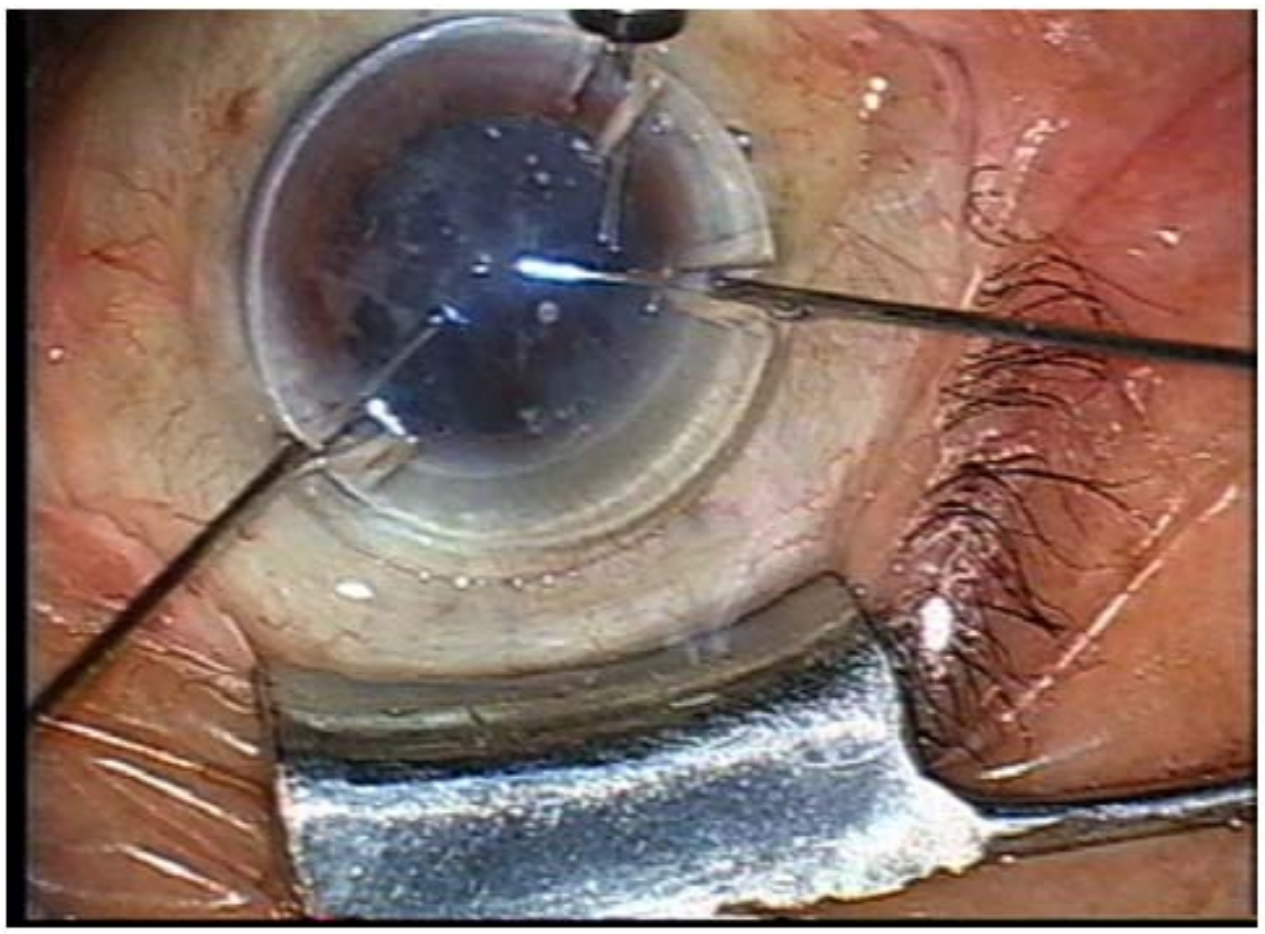

B. Notched contact lens used in 25-gauge transcorneal vitrectomy. 


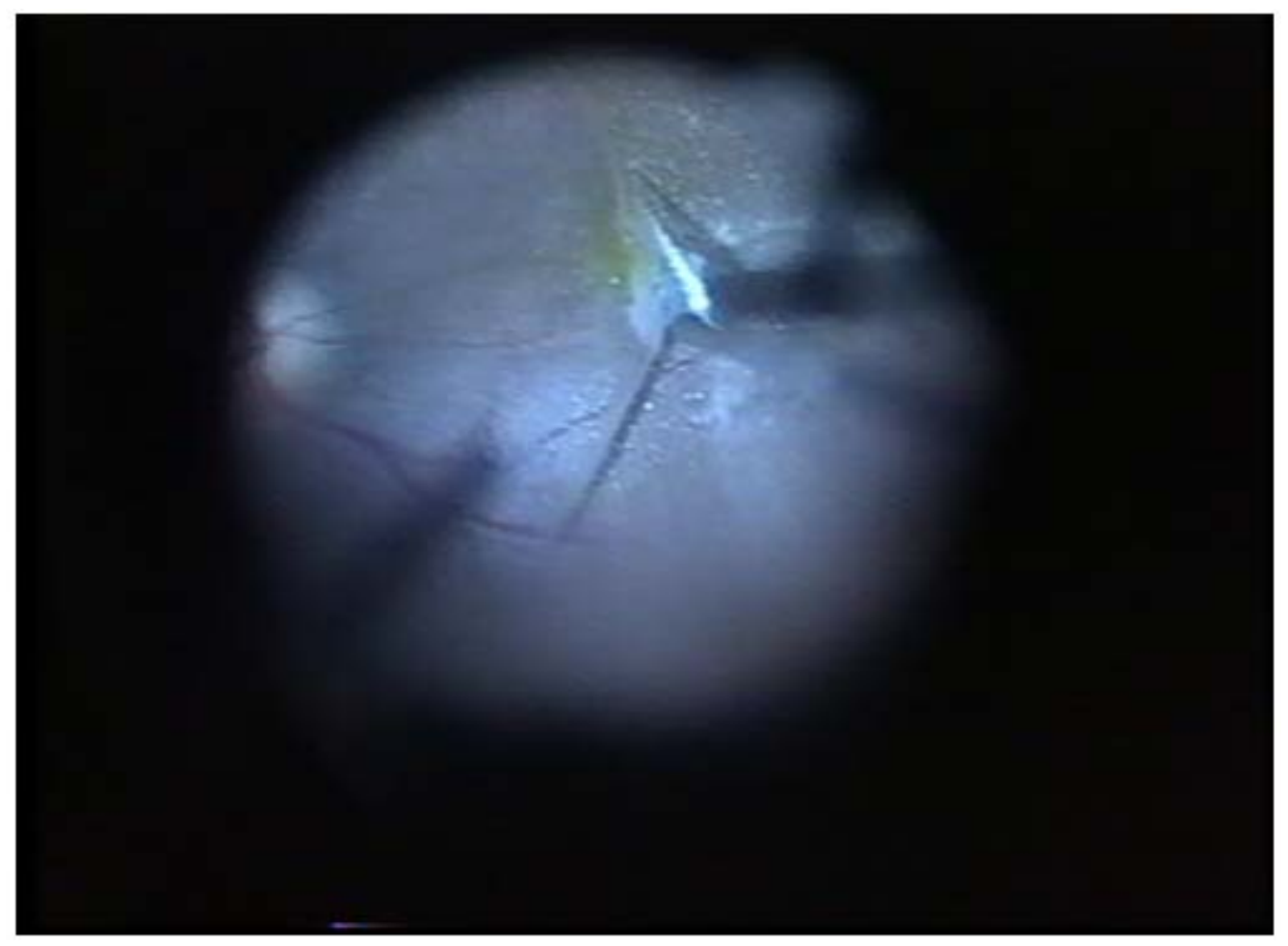

C. ERM peeling.

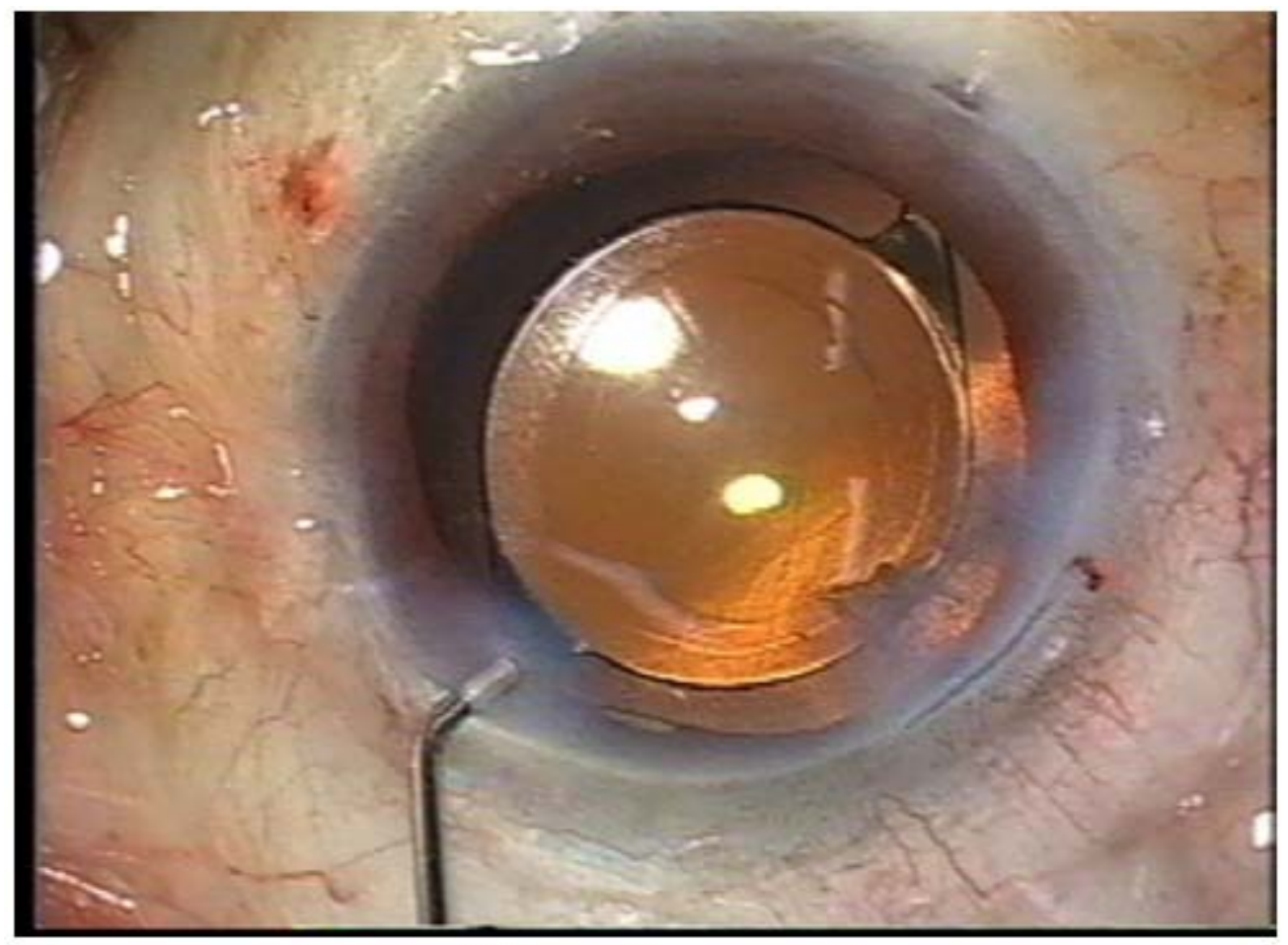

D. Insertion of intraocular lens into capsular bag after vitrectomy. 
Table 1. Clinical summaries of patients.

\begin{tabular}{cccccccccc}
\hline \multicolumn{1}{c}{ Case No. Age Sex } & Eye & Cause & Otherfactors & Complications & $\begin{array}{c}\text { Visual acuity } \\
\text { (pre-op) }\end{array}$ & $\begin{array}{c}\text { Visual acuity } \\
\text { (1 Mn post-op) }\end{array}$ & $\begin{array}{c}\text { Visual acuity } \\
\text { (6 Mn post-op) }\end{array}$ \\
\hline 1 & 72 & F & R & Post RD op & Posterior capsule rupture & None & $20 / 80$ & $20 / 25$ & $20 / 20$ \\
2 & 39 & M & R & BRvo & Giant bleb post trabeculectomy & None & $20 / 80$ & $20 / 160$ & $20 / 100$ \\
3 & 62 & F & L & Retinal tears & None & $20 / 80$ & $20 / 40$ & $20 / 16$ \\
4 & 77 & M & R & Idiopathic & None & $20 / 300$ & $20 / 25$ & $20 / 25$ \\
5 & 66 & F & L & Idiopathic & None & $20 / 50$ & $20 / 25$ & $20 / 25$ \\
6 & 70 & F & L & Idiopathic & Iatrogeniclamellar & $20 / 40$ & $20 / 200$ & $20 / 100$ \\
7 & 77 & M & R & Idiopathic & macularhole & None & $20 / 80$ & $20 / 50$ & $20 / 20$ \\
\hline
\end{tabular}

F, female; $M$, male; $R$, right; $L$, left; $M n$, months; $R D$, retinal detachment; BRVO, branch retinal vein occlusion; pre-op, before surgery; post-op, after surgery. 